\title{
Numerical Solution for Fuzzy Diffusion Problem via Two Parameter Alternating Group Explicit Technique
}

\author{
A. A. Dahalan ${ }^{1, *}$, J. Sulaiman ${ }^{2}$ \\ ${ }^{1}$ Department of Mathematics, Centre for Defence Foundation Studies, National Defence University of Malaysia, Malaysia \\ ${ }^{2}$ Faculty of Science and Natural Resources, Universiti Malaysia Sabah, Malaysia
}

Received November 26, 2020; Revised January 21, 2021; Accepted March 24, 2021

\begin{abstract}
Cite This Paper in the following Citation Styles
(a): [1] A. A. Dahalan, J. Sulaiman, "Numerical Solution for Fuzzy Diffusion Problem via Two Parameter Alternating Group Explicit Technique," Mathematics and Statistics, Vol. 9, No. 2, pp. 98 - 105, 2021. DOI: 10.13189/ms.2021.090203.
\end{abstract}

(b): A. A. Dahalan, J. Sulaiman (2021). Numerical Solution for Fuzzy Diffusion Problem via Two Parameter Alternating Group Explicit Technique. Mathematics and Statistics, 9(2), 98 - 105. DOI: 10.13189/ms.2021.090203.

Copyright $(2021$ by authors, all rights reserved. Authors agree that this article remains permanently open access under the terms of the Creative Commons Attribution License 4.0 International License

Abstract The computational technique has become a significant area of study in physics and engineering. The first method to evaluate the problems numerically was a finite difference. In 2002, a computational approach, an explicit finite difference technique, was used to overcome the fuzzy partial differential equation (FPDE) based on the Seikkala derivative. The application of the iterative technique, in particular the Two Parameter Alternating Group Explicit (TAGE) method, is employed to resolve the finite difference approximation resulting after the fuzzy heat equation is investigated in this article. This article broadens the use of the TAGE iterative technique to solve fuzzy problems due to the reliability of the approaches. The development and execution of the TAGE technique towards the full-sweep (FS) and half-sweep (HS) techniques are also presented. The idea of using the HS scheme is to reduce the computational complexity of the iterative methods by nearly/more than half. Additionally, numerical outcomes from the solution of two experimental problems are included and compared with the Alternating Group Explicit (AGE) approaches to clarify their feasibility. In conclusion, the families of the TAGE technique have been used to overcome the linear system structure through a one-dimensional fuzzy diffusion (1D-FD) discretization using a finite difference scheme. The findings suggest that the HSTAGE approach is surpassing in terms of iteration counts, time taken, and Hausdorff distance relative to the FSTAGE and AGE approaches. It demonstrates that the number of iterations for HSTAGE approach has decreased by approximately $71.60-72.95 \%$, whereas for the execution time, the implementation of HSTAGE method is between $74.05-86.42 \%$ better. Since TAGE is ideal for concurrent processing, this method has been seen as the key benefit as it consumes sets of independent tasks that can be performed at the same time. The ability of the suggested technique is projected to be useful for the advanced exploration in solving any multi-dimensional FPDEs.

Keywords Fuzzy Heat Equation, Implicit Scheme, Two-Stage Iteration, One-Dimensional, Finite Difference Method, Half-Sweep

\section{Introduction}

The computational practice has progressed significantly in the past 40 years, despite the advances made in computer technology. The numerical approach has become an essential field in the area of research in physics and engineering. The earliest method among the most crucial techniques for numerically analyzing the practical problems was a finite difference. The finite difference scheme employs divided difference expressions developed from the local Taylor series to substitute differential or partial operators. [1] was the first to introduce the notion of the fuzzy derivative. Although, the one who described and applied the extension theory was [2]. The equations of fuzzy differential and the problem of fuzzy initial value have frequently been addressed [3-6]. In [7,8], a computational approach, an explicit finite difference 
technique, is used to overcome a fuzzy partial differential equation (FPDE) based on the Seikkala derivative. Typically, it is exceedingly difficult to calculate the solution of FPDEs. The exact solution to this complication can only be sought in certain particular situations.

Alternating Group Explicit (AGE) approach has been demonstrated to remain an important as well as an efficient tool since it was developed. Evans and Sahimi [9] illustrates the extended AGE technique for problems involving both parabolic and hyperbolic PDEs. In this approach, however, only one parameter has been taken into account. Later, this technique has also been expanded by [10], through the implementation of the Two Parameter Alternating Group Explicit (TAGE) technique, which verified its better than the AGE scheme.

The one-dimensional fuzzy diffusion (1D-FD) equation will be numerically solved using the second-order central finite difference approximation to discretize 1D-FD in linear systems based on the derivative of Seikkala [6]. The generated linear systems are then resolved by the TAGE method iteratively. Previous studies associated to the TAGE iterative technique and its modification [11-13] have demonstrated that this approach was used extensively to unravel the problems in non-fuzzy cases. This article widens the use of the TAGE iterative approach to solve fuzzy issues because of the efficiency of the methods.

The iterative technique turns out to be a natural alternative for obtaining a solution to the fuzzy numerical problem since the fuzzy linear systems exist. Finding towards the comprehension to the half-sweep iterative technique is motivated by an earlier analysis of the Explicit Decoupled Group scheme [14]. The main feature of this concept is the methodology of reducing the computational complexity. The finite grid (see Fig. 1) is used to elucidate the construction of the equations for fulland half-sweep difference approximation. Applications of these projected iterative approaches to the inner solid nodal points shall be carried out until the convergence test has been identified. In the meantime, the approximation solutions can be determined using the direct method for the remaining points $[14,15]$.

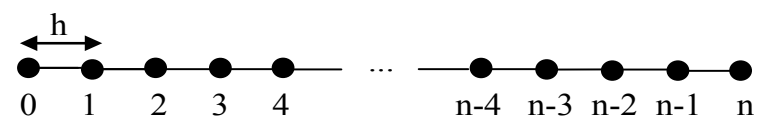

(a)

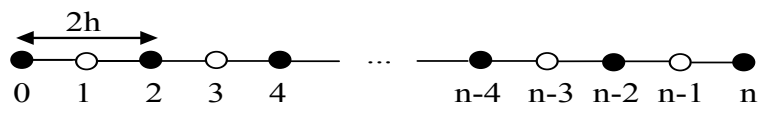

(b)

Figure 1. The arrangement of solid nodal points for full- (a) and half-sweep (b) cases

\section{Methodology}

For the real numbers, let $\tilde{U}$ be a fuzzy subset. As part of the membership function, the said fuzzy subset should be described as $\tilde{U}(x)$ calculated at $x$ for a number in $[0,1]$. An $\alpha$-cut of the fuzzy subset (with $\alpha=$ crisp number) is symbolized by $\tilde{U}(\alpha)$ and then defined with $I=\{(x, t) \mid 0 \leq x \leq 1,0 \leq t \leq T\}$. The intervals for all crisp fuzzy subsets shall be shown by $\tilde{U}(\alpha)=[\underline{U}(\alpha), \bar{U}(\alpha)]$. This is due to the fuzzy numbers $\alpha$-cut are continuously closed and bounded [7]. Presuming the parametric system of the fuzzy function $U$ is $(\underline{U}, \bar{U})$.

Considering the equation of fuzzy diffusion as follows

$$
\frac{\partial \tilde{U}}{\partial t}(x, t)-\lambda \frac{\partial^{2} \tilde{U}}{\partial x^{2}}(x, t)=0, \quad 0<x<l, t>0 .
$$

Let $R=\{(x, t) \mid(x, t) \in I\}$, while $S$ represent the boundary of $R$. The subsequent issues will be solved numerically:

$$
\begin{aligned}
& \frac{\partial \underline{U}}{\partial t}(x, t ; \alpha)-\lambda \frac{\partial^{2} \underline{U}}{\partial x^{2}}(x, t ; \alpha)=0, \\
& \underline{U}(0, t ; \alpha)=\underline{U}(l, t ; \alpha)=0, \quad t>0, \\
& \underline{U}(x, 0 ; \alpha)=\underline{f}(x ; \alpha), \quad 0 \leq x \leq l
\end{aligned}
$$

and

$$
\begin{aligned}
& \frac{\partial \bar{U}}{\partial t}(x, t ; \alpha)-\lambda \frac{\partial^{2} \bar{U}}{\partial x^{2}}(x, t ; \alpha)=0, \\
& \bar{U}(0, t ; \alpha)=\bar{U}(l, t ; \alpha)=0, \quad t>0, \\
& \bar{U}(x, 0 ; \alpha)=\bar{f}(x ; \alpha), \quad 0 \leq x \leq l
\end{aligned}
$$

for $(x, t) \in S$. The region $[0,1] \times[0, T]$ is divided within the mesh of the $M \times N$ over dimensional step size. For $x$-axis, the step size of $h=\frac{1}{N}$ (with $\left.x_{i}=i h(i=0,1,2, \ldots, N)\right)$. And for $t$-direction, the step size is $k=\frac{T}{M} \quad$ (with $t_{j}=j k(i=0,1,2, \ldots, M)$ ). Applying the implicit discretization practice to such issues would therefore yield

$$
\begin{aligned}
& \frac{\partial \underline{\underline{U}}}{\partial t} \approx \frac{U_{i, j+1}-U_{i, j}}{k}, \\
& \frac{\partial \bar{U}}{\partial t} \approx \frac{\overline{U_{i, j+1}}-\overline{U_{i, j}}}{k},
\end{aligned}
$$


and

$$
\begin{aligned}
& \frac{\partial^{2} \underline{U}}{\partial x^{2}} \approx \lambda\left[\frac{U_{i-1, j+1}-2 U_{i, j+1}+\underline{U_{i+1, j+1}}}{h^{2}}\right],
\end{aligned}
$$

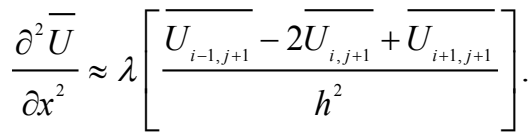

Via consuming equations (3a) and (4a), equations (2a) able to signified as

$$
\begin{aligned}
\left(\frac{k \lambda}{h^{2}}\right)_{\underline{U_{i-1, j+1}}}-( & \left.\left(\frac{k \lambda}{h^{2}}\right)+1\right) \underline{U_{i, j+1}} \\
& +\left(\frac{k \lambda}{h^{2}}\right) \underline{U}_{i+1, j+1}=-U_{i, j} .
\end{aligned}
$$

Whereas, by replacing equations (3b) and (4b) towards equation $(2 b)$, presented as

$$
\begin{aligned}
\left(\frac{k \lambda}{h^{2}}\right) \overline{U_{i-1, j+1}}-\left(2\left(\frac{k \lambda}{h^{2}}\right)+1\right) \overline{U_{i, j+1}} & \\
& +\left(\frac{k \lambda}{h^{2}}\right) \overline{U_{i+1, j+1}}=-\overline{U_{i, j}}
\end{aligned}
$$

for $i=1,2, \ldots, N-1$ and $j=1,2, \ldots, M-1$. With the exception that, grounded on the $\alpha$-cuts interval, both equations (5a) and (5b) possess the identical type of equation, except the variations, are found solely at the upper and lower boundary. Therefore, this may then be simplified as follows

$$
-\beta U_{i-1, j+1}+(2 \beta+1) U_{i, j+1}-\beta U_{i+1, j+1}=U_{i, j}
$$

where $\beta=\left(\frac{k \lambda}{h^{2}}\right)$. Simplification of $(6)$ in the form of a matrix is referred to as

$$
A \tilde{U}_{j+1}=\tilde{b}_{j} .
$$

\section{Design of Two Parameter Alternating Group Explicit Scheme}

Following the growth of the AGE method, the TAGE iterative method is one of the families of the AGE technique. In fact, it can be easily shown that the distinction between these methods lies entirely in the use of weighted parameter $r$. The AGE technique consists of a single parameter, $r_{1}$. Whereas the TAGE technique contains two parameters, $r_{1}$ and $r_{2}$, each of which is used for the calculation of the first and second sweeps, respectively. It should be emphasized that if both parameters have the same value, i.e. $r_{1}=r_{2}$, then TAGE can be reduced as the AGE method. This makes it easier to discuss, and this section will only focus on the TAGE iterative approach.

On the basis of prior study, hypothetically $[12,13,16,17]$ have explored how the value of parameter $r$ can be determined. Herein, the optimal value for parameters $r_{1}$ and $r_{2}$ are measured by employing numerous computational experiment so that when the iteration number is lesser, the optimal value is found.

TAGE categories can be regarded as a two-step technique in solving a linear system. Not any of the researchers had tried to use this approach, particularly in addressing fuzzy problems initiated by the discretization of FPDE. The implementation of this scheme would resolve the fuzzy linear system as stated in equation (1). Following the partitioning of matrix $A$ by the sum of its constitutive symmetrical and positive definite matrices $[10,18]$, matrix $A$ in equation (7) can be stated as

$$
A=G_{1}+G_{2}
$$

whereby
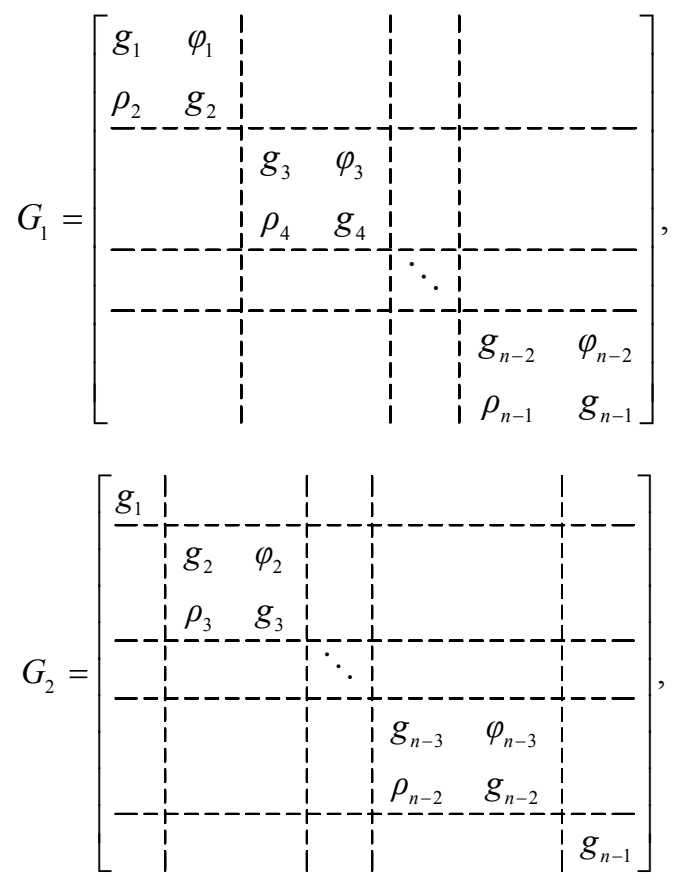

for $n$ is an odd number. Likewise, if $n$ is an even number

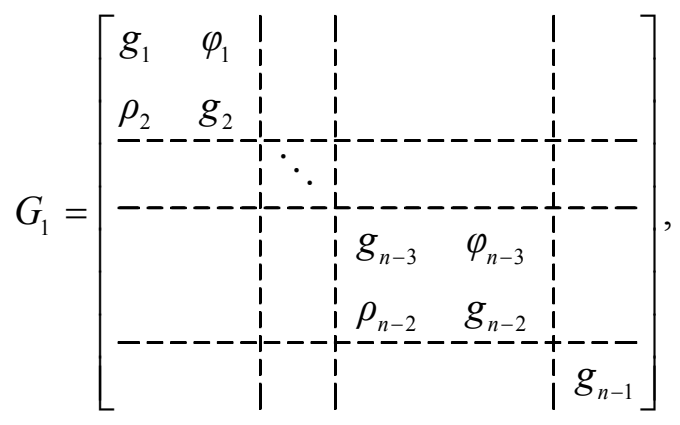




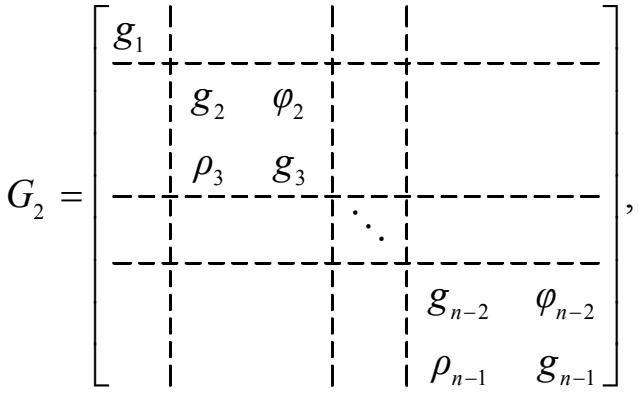

where $g_{i}=\frac{\beta_{i}}{2}(i=1,2, \ldots, n-1)$. This article simply analyzes for the case $n$ is even.

Then equation (7) becomes

$$
\left(G_{1}+G_{2}\right) \tilde{U}=b .
$$

As a result, the explicit form of the TAGE scheme is composed as

$$
\begin{aligned}
& \tilde{U}^{\left(k+\frac{1}{2}\right)}=\left(G_{1}+r_{1} I\right)^{-1}\left[b-\left(G_{2}-r_{1} I\right) \tilde{U}^{(k)}\right], \\
& \tilde{U}^{(k+1)}=\left(G_{2}+r_{2} I\right)^{-1}\left[b-\left(G_{1}-r_{2} I\right) \tilde{U}^{\left(k+\frac{1}{2}\right)}\right],
\end{aligned}
$$

where $r_{1}, r_{2}>0$ are the parameters of acceleration, and duo of $\left(G_{1}+r_{1} I\right)$ and $\left(G_{2}+r_{2} I\right)$ are invertible. Therefore, based on equations (10a) and (10b), the execution of the families of TAGE approaches is demonstrated as in Algorithm 1.

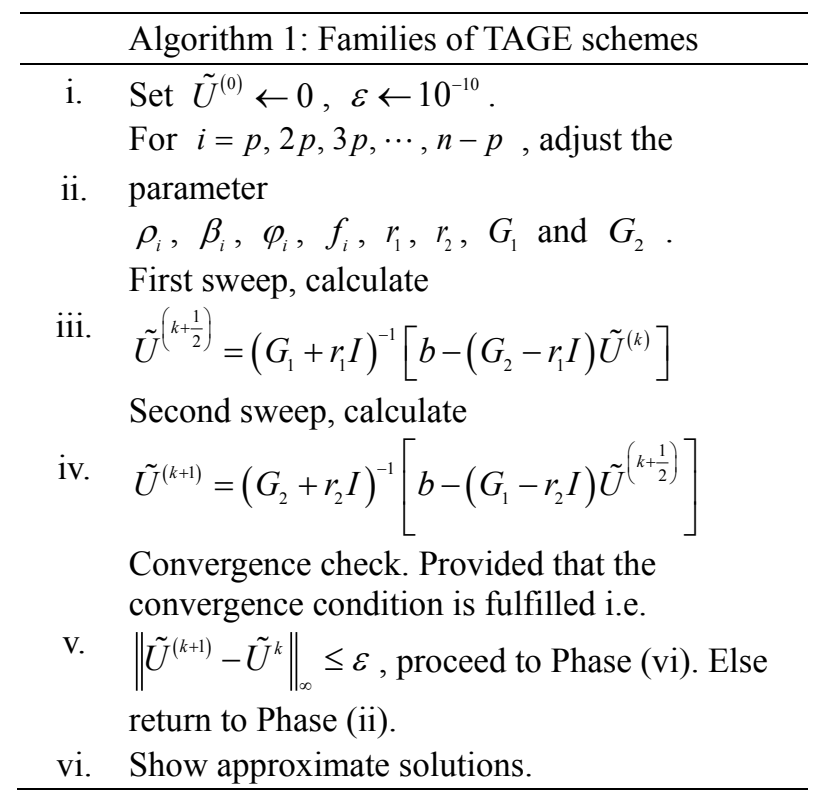

\section{Numerical Experiments}

With the intention of verifying the efficiency of AGE, FSTAGE, and HSTAGE techniques, two problems of 1D-FD are considered. Three parameters, namely iteration counts, time taken (in seconds), and Hausdorff distance (as stated in Definition 1), are observed for comparative purposes. In Tables 1 to 5, the numerical performances of the suggested approaches for different values of $\alpha$ are

\begin{tabular}{|c|c|c|c|c|c|c|c|}
\hline & & \multirow{2}{*}{ Methods } & \multicolumn{5}{|c|}{$n$} \\
\hline & & & 512 & 1024 & 2048 & 4096 & 8192 \\
\hline \multirow{9}{*}{$\begin{array}{c}\text { Example } \\
1\end{array}$} & \multirow{3}{*}{$\begin{array}{c}\text { Iteration } \\
\text { counts }\end{array}$} & AGE & 5199 & 18926 & 67947 & 239878 & 829284 \\
\hline & & FSTAGE & 5001 & 18324 & 65958 & 235952 & 829284 \\
\hline & & HSTAGE & 1357 & 5001 & 18324 & 65958 & 235952 \\
\hline & \multirow{3}{*}{ Time taken } & AGE & 9.80 & 71.25 & 511.46 & 3622.74 & 25049.26 \\
\hline & & FSTAGE & 9.45 & 68.93 & 495.66 & 3549.95 & 24977.48 \\
\hline & & HSTAGE & 1.31 & 9.36 & 68.32 & 498.01 & 3716.55 \\
\hline & \multirow{3}{*}{$\begin{array}{l}\text { Hausdorff } \\
\text { distance }\end{array}$} & AGE & $4.7013 \mathrm{e}-04$ & $4.6910 \mathrm{e}-04$ & $4.6513 \mathrm{e}-04$ & $4.4929 \mathrm{e}-04$ & $3.8569 \mathrm{e}-04$ \\
\hline & & FSTAGE & $4.7034 \mathrm{e}-04$ & $4.6968 \mathrm{e}-04$ & $4.6736 \mathrm{e}-04$ & $4.5317 \mathrm{e}-04$ & $3.8569 \mathrm{e}-04$ \\
\hline & & HSTAGE & $4.7060 \mathrm{e}-04$ & $4.7034 \mathrm{e}-04$ & $4.6968 \mathrm{e}-04$ & $4.6736 \mathrm{e}-04$ & $4.5317 \mathrm{e}-04$ \\
\hline \multirow{9}{*}{$\begin{array}{c}\text { Example } \\
2\end{array}$} & \multirow{3}{*}{$\begin{array}{c}\text { Iteration } \\
\text { counts }\end{array}$} & AGE & 1749 & 6462 & 23816 & 87009 & 314488 \\
\hline & & FSTAGE & 1747 & 6458 & 23816 & 87009 & 314488 \\
\hline & & HSTAGE & 484 & 1747 & 6458 & 23814 & 87009 \\
\hline & \multirow{3}{*}{ Time taken } & AGE & 14.74 & 67.42 & 338.32 & 2180.24 & 13353.35 \\
\hline & & FSTAGE & 14.72 & 66.27 & 334.78 & 2058.80 & 13245.96 \\
\hline & & HSTAGE & 3.69 & 14.77 & 66.63 & 336.86 & 2127.83 \\
\hline & \multirow{3}{*}{$\begin{array}{l}\text { Hausdorff } \\
\text { distance }\end{array}$} & AGE & $3.7252 \mathrm{e}-03$ & $3.7232 \mathrm{e}-03$ & $3.7198 \mathrm{e}-03$ & $3.7071 \mathrm{e}-03$ & $3.6561 \mathrm{e}-03$ \\
\hline & & FSTAGE & $3.7253 \mathrm{e}-03$ & $3.7234 \mathrm{e}-03$ & $3.7198 \mathrm{e}-03$ & $3.7071 \mathrm{e}-03$ & $3.6561 \mathrm{e}-03$ \\
\hline & & HSTAGE & $3.7301 \mathrm{e}-03$ & $3.7253 \mathrm{e}-03$ & $3.7234 \mathrm{e}-03$ & $3.7197 \mathrm{e}-03$ & $3.7071 \mathrm{e}-03$ \\
\hline
\end{tabular}
documented on the basis of these two examples.

Table 1. The performance of three terms amongst AGE, FSTAGE, and HSTAGE approaches at $\alpha=0.00$ 
Table 2. The performance of three terms amongst AGE, FSTAGE, and HSTAGE approaches at $\alpha=0.25$.

\begin{tabular}{|c|c|c|c|c|c|c|c|}
\hline & & \multirow{2}{*}{ Methods } & \multicolumn{5}{|c|}{$n$} \\
\hline & & & 512 & 1024 & 2048 & 4096 & 8192 \\
\hline \multirow{9}{*}{$\begin{array}{c}\text { Example } \\
1\end{array}$} & \multirow{3}{*}{$\begin{array}{l}\text { Iteration } \\
\text { counts }\end{array}$} & AGE & 5224 & 19026 & 68348 & 241494 & 835853 \\
\hline & & FSTAGE & 5024 & 18418 & 66337 & 237521 & 835853 \\
\hline & & HSTAGE & 1363 & 5024 & 18418 & 66337 & 237521 \\
\hline & \multirow{3}{*}{ Time taken } & AGE & 9.86 & 71.60 & 515.27 & 3644.49 & 25184.00 \\
\hline & & FSTAGE & 9.47 & 69.24 & 498.36 & 3574.98 & 25171.99 \\
\hline & & HSTAGE & 1.31 & 9.41 & 68.73 & 500.84 & 3583.61 \\
\hline & \multirow{3}{*}{$\begin{array}{l}\text { Hausdorff } \\
\text { distance }\end{array}$} & AGE & $4.3092 \mathrm{e}-04$ & $4.2990 \mathrm{e}-04$ & $4.2593 \mathrm{e}-04$ & $4.1009 \mathrm{e}-04$ & $3.4650 \mathrm{e}-04$ \\
\hline & & FSTAGE & $4.3113 \mathrm{e}-04$ & $4.3048 \mathrm{e}-04$ & $4.2814 \mathrm{e}-04$ & $4.1394 \mathrm{e}-04$ & $3.4650 \mathrm{e}-04$ \\
\hline & & HSTAGE & $4.3139 \mathrm{e}-04$ & $4.3113 \mathrm{e}-04$ & $4.3048 \mathrm{e}-04$ & $4.2814 \mathrm{e}-04$ & $4.1394 \mathrm{e}-04$ \\
\hline \multirow{9}{*}{$\begin{array}{c}\text { Example } \\
2\end{array}$} & \multirow{3}{*}{$\begin{array}{c}\text { Iteration } \\
\text { counts }\end{array}$} & AGE & 1756 & 6488 & 23920 & 87430 & 316172 \\
\hline & & FSTAGE & 1754 & 6484 & 23920 & 87430 & 316172 \\
\hline & & HSTAGE & 486 & 1754 & 6484 & 23919 & 87430 \\
\hline & \multirow{3}{*}{ Time taken } & AGE & 14.77 & 67.42 & 339.34 & 2097.65 & 13897.57 \\
\hline & & FSTAGE & 14.77 & 66.57 & 337.35 & 2075.37 & 13603.57 \\
\hline & & HSTAGE & 3.77 & 14.77 & 66.80 & 339.32 & 2144.81 \\
\hline & \multirow{3}{*}{$\begin{array}{l}\text { Hausdorff } \\
\text { distance }\end{array}$} & AGE & $3.4147 \mathrm{e}-03$ & $3.4129 \mathrm{e}-03$ & $3.4095 \mathrm{e}-03$ & $3.3967 \mathrm{e}-03$ & $3.3457 \mathrm{e}-03$ \\
\hline & & FSTAGE & $3.4148 \mathrm{e}-03$ & $3.4130 \mathrm{e}-03$ & $3.4095 \mathrm{e}-03$ & $3.3967 \mathrm{e}-03$ & $3.3457 \mathrm{e}-03$ \\
\hline & & HSTAGE & $3.4193 \mathrm{e}-03$ & $3.4148 \mathrm{e}-03$ & $3.4130 \mathrm{e}-03$ & $3.4094 \mathrm{e}-03$ & $3.3967 \mathrm{e}-03$ \\
\hline
\end{tabular}

Table 3. The performance of three terms amongst AGE, FSTAGE, and HSTAGE approaches at $\alpha=0.50$.

\begin{tabular}{|c|c|c|c|c|c|c|c|}
\hline & & \multirow{2}{*}{ Methods } & \multicolumn{5}{|c|}{$n$} \\
\hline & & & 512 & 1024 & 2048 & 4096 & 8192 \\
\hline \multirow{9}{*}{$\begin{array}{c}\text { Example } \\
1\end{array}$} & \multirow{3}{*}{$\begin{array}{c}\text { Iteration } \\
\text { counts }\end{array}$} & AGE & 5240 & 19090 & 68605 & 242527 & 840044 \\
\hline & & FSTAGE & 5039 & 18479 & 66579 & 238524 & 840044 \\
\hline & & HSTAGE & 1369 & 5039 & 18479 & 66579 & 238524 \\
\hline & \multirow{3}{*}{ Time taken } & AGE & 9.89 & 71.87 & 516.64 & 3662.89 & 25503.54 \\
\hline & & FSTAGE & 9.50 & 69.53 & 500.39 & 3587.95 & 25307.30 \\
\hline & & HSTAGE & 1.32 & 9.44 & 68.98 & 503.22 & 3594.38 \\
\hline & \multirow{3}{*}{$\begin{array}{l}\text { Hausdorff } \\
\text { distance }\end{array}$} & AGE & $3.9172 \mathrm{e}-04$ & $3.9070 \mathrm{e}-04$ & $3.8673 \mathrm{e}-04$ & $3.7089 \mathrm{e}-04$ & $3.0730 \mathrm{e}-04$ \\
\hline & & FSTAGE & $3.9193 \mathrm{e}-04$ & $3.9127 \mathrm{e}-04$ & $3.8892 \mathrm{e}-04$ & $3.7471 \mathrm{e}-04$ & $3.0730 \mathrm{e}-04$ \\
\hline & & HSTAGE & $3.9217 \mathrm{e}-04$ & $3.9193 \mathrm{e}-04$ & $3.9127 \mathrm{e}-04$ & $3.8892 \mathrm{e}-04$ & $3.7471 \mathrm{e}-04$ \\
\hline \multirow{9}{*}{$\begin{array}{c}\text { Example } \\
2\end{array}$} & \multirow{3}{*}{$\begin{array}{l}\text { Iteration } \\
\text { counts }\end{array}$} & AGE & 1760 & 6505 & 23987 & 87698 & 317249 \\
\hline & & FSTAGE & 1758 & 6500 & 23987 & 87698 & 317249 \\
\hline & & HSTAGE & 487 & 1758 & 6500 & 23986 & 87698 \\
\hline & \multirow{3}{*}{ Time taken } & AGE & 14.79 & 68.09 & 341.25 & 2064.06 & 13454.90 \\
\hline & & FSTAGE & 14.72 & 66.69 & 337.88 & 2058.49 & 13448.08 \\
\hline & & HSTAGE & 3.82 & 14.85 & 66.98 & 339.70 & 2130.18 \\
\hline & \multirow{3}{*}{$\begin{array}{l}\text { Hausdorff } \\
\text { distance }\end{array}$} & AGE & $3.1043 e-03$ & $3.1025 \mathrm{e}-03$ & $3.0991 \mathrm{e}-03$ & $3.0864 \mathrm{e}-03$ & $3.0354 \mathrm{e}-03$ \\
\hline & & FSTAGE & $3.1044 \mathrm{e}-03$ & $3.1026 \mathrm{e}-03$ & $3.0991 \mathrm{e}-03$ & $3.0864 \mathrm{e}-03$ & $3.0354 \mathrm{e}-03$ \\
\hline & & HSTAGE & $3.1084 \mathrm{e}-03$ & $3.1044 \mathrm{e}-03$ & $3.1026 \mathrm{e}-03$ & $3.0991 \mathrm{e}-03$ & $3.0864 \mathrm{e}-03$ \\
\hline
\end{tabular}


Table 4. The performance of three terms amongst AGE, FSTAGE, and HSTAGE approaches at $\alpha=0.75$.

\begin{tabular}{|c|c|c|c|c|c|c|c|}
\hline & & \multirow{2}{*}{ Methods } & \multicolumn{5}{|c|}{$n$} \\
\hline & & & 512 & 1024 & 2048 & 4096 & 8192 \\
\hline \multirow{9}{*}{$\begin{array}{c}\text { Example } \\
1\end{array}$} & \multirow{3}{*}{$\begin{array}{c}\text { Iteration } \\
\text { counts }\end{array}$} & AGE & 5248 & 19125 & 68749 & 243106 & 842391 \\
\hline & & FSTAGE & 5047 & 18512 & 66715 & 239086 & 842391 \\
\hline & & HSTAGE & 1371 & 5047 & 18512 & 66715 & 239086 \\
\hline & \multirow{3}{*}{ Time taken } & AGE & 9.91 & 71.98 & 517.61 & 3673.02 & 25372.88 \\
\hline & & FSTAGE & 9.52 & 69.59 & 501.06 & 3599.82 & 25371.09 \\
\hline & & HSTAGE & 1.33 & 9.47 & 69.04 & 504.17 & 3598.52 \\
\hline & \multirow{3}{*}{$\begin{array}{l}\text { Hausdorff } \\
\text { distance }\end{array}$} & AGE & $3.5251 \mathrm{e}-04$ & $3.5150 \mathrm{e}-04$ & $3.4753 \mathrm{e}-04$ & $3.3169 \mathrm{e}-04$ & $2.6810 \mathrm{e}-04$ \\
\hline & & FSTAGE & $3.5272 \mathrm{e}-04$ & $3.5206 \mathrm{e}-04$ & $3.4970 \mathrm{e}-04$ & $3.3548 \mathrm{e}-04$ & $2.6810 \mathrm{e}-04$ \\
\hline & & HSTAGE & $3.5295 \mathrm{e}-04$ & $3.5272 \mathrm{e}-04$ & $3.5206 \mathrm{e}-04$ & $3.4970 \mathrm{e}-04$ & $3.3548 \mathrm{e}-04$ \\
\hline \multirow{9}{*}{$\begin{array}{c}\text { Example } \\
2\end{array}$} & \multirow{3}{*}{$\begin{array}{c}\text { Iteration } \\
\text { counts }\end{array}$} & AGE & 1762 & 6514 & 24025 & 87849 & 317854 \\
\hline & & FSTAGE & 1760 & 6509 & 24025 & 87849 & 317854 \\
\hline & & HSTAGE & 487 & 1760 & 6509 & 24023 & 87849 \\
\hline & \multirow{3}{*}{ Time taken } & AGE & 15.02 & 67.86 & 352.58 & 2132.63 & 14071.04 \\
\hline & & FSTAGE & 14.87 & 66.67 & 338.05 & 2076.88 & 13480.32 \\
\hline & & HSTAGE & 3.74 & 14.90 & 67.33 & 339.94 & 2134.84 \\
\hline & \multirow{3}{*}{$\begin{array}{l}\text { Hausdorff } \\
\text { distance }\end{array}$} & AGE & $2.7938 \mathrm{e}-03$ & $2.7921 \mathrm{e}-03$ & $2.7888 \mathrm{e}-03$ & $2.7761 \mathrm{e}-03$ & $2.7251 \mathrm{e}-03$ \\
\hline & & FSTAGE & $2.7939 \mathrm{e}-03$ & $2.7923 \mathrm{e}-03$ & $2.7888 \mathrm{e}-03$ & $2.7761 \mathrm{e}-03$ & $2.7251 \mathrm{e}-03$ \\
\hline & & HSTAGE & $2.7976 \mathrm{e}-03$ & $2.7939 \mathrm{e}-03$ & $2.7923 \mathrm{e}-03$ & $2.7887 \mathrm{e}-03$ & $2.7761 \mathrm{e}-03$ \\
\hline
\end{tabular}

Table 5. The performance of three terms amongst AGE, FSTAGE, and HSTAGE approaches at $\alpha=1.00$

\begin{tabular}{|c|c|c|c|c|c|c|c|}
\hline & & \multirow{2}{*}{ Methods } & \multicolumn{5}{|c|}{$n$} \\
\hline & & & 512 & 1024 & 2048 & 4096 & 8192 \\
\hline \multirow{9}{*}{$\begin{array}{c}\text { Example } \\
1\end{array}$} & \multirow{3}{*}{$\begin{array}{c}\text { Iteration } \\
\text { counts }\end{array}$} & AGE & 5251 & 19137 & 68795 & 243293 & 843148 \\
\hline & & FSTAGE & 5050 & 18523 & 66759 & 239267 & 843148 \\
\hline & & HSTAGE & 1372 & 5052 & 18523 & 66759 & 239267 \\
\hline & \multirow{3}{*}{ Time taken } & AGE & 9.91 & 72.07 & 518.22 & 3677.87 & 25393.35 \\
\hline & & FSTAGE & 9.53 & 69.66 & 501.69 & 3600.26 & 25392.15 \\
\hline & & HSTAGE & 1.31 & 9.45 & 69.10 & 504.65 & 3602.47 \\
\hline & \multirow{3}{*}{$\begin{array}{l}\text { Hausdorff } \\
\text { distance }\end{array}$} & AGE & $3.1331 \mathrm{e}-04$ & $3.1230 \mathrm{e}-04$ & $3.0833 \mathrm{e}-04$ & $2.9249 \mathrm{e}-04$ & $2.2890 \mathrm{e}-04$ \\
\hline & & FSTAGE & $3.1351 \mathrm{e}-04$ & $3.1285 \mathrm{e}-04$ & $3.1047 \mathrm{e}-04$ & $2.9624 \mathrm{e}-04$ & $2.2890 \mathrm{e}-04$ \\
\hline & & HSTAGE & $3.1373 e-04$ & $3.1349 \mathrm{e}-04$ & $3.1285 \mathrm{e}-04$ & $3.1047 \mathrm{e}-04$ & $2.9625 \mathrm{e}-04$ \\
\hline \multirow{9}{*}{$\begin{array}{c}\text { Example } \\
2\end{array}$} & \multirow{3}{*}{$\begin{array}{c}\text { Iteration } \\
\text { counts }\end{array}$} & AGE & 1763 & 6517 & 24037 & 87897 & 318049 \\
\hline & & FSTAGE & 1761 & 6512 & 24037 & 87897 & 318049 \\
\hline & & HSTAGE & 487 & 1761 & 6512 & 24036 & 87897 \\
\hline & \multirow{3}{*}{ Time taken } & AGE & 15.06 & 67.94 & 357.77 & 2132.47 & 13916.13 \\
\hline & & FSTAGE & 14.84 & 66.83 & 338.23 & 2091.35 & 13624.88 \\
\hline & & HSTAGE & 3.78 & 14.79 & 67.30 & 340.42 & 2110.91 \\
\hline & \multirow{3}{*}{$\begin{array}{l}\text { Hausdorff } \\
\text { distance }\end{array}$} & AGE & $2.4834 \mathrm{e}-03$ & $2.4818 \mathrm{e}-03$ & $2.4785 \mathrm{e}-03$ & $2.4658 \mathrm{e}-03$ & $2.4148 \mathrm{e}-03$ \\
\hline & & FSTAGE & $2.4834 \mathrm{e}-03$ & $2.4819 \mathrm{e}-03$ & $2.4785 \mathrm{e}-03$ & $2.4878 \mathrm{e}-03$ & $2.4148 \mathrm{e}-03$ \\
\hline & & HSTAGE & $2.4867 \mathrm{e}-03$ & $2.4834 \mathrm{e}-03$ & $2.4819 \mathrm{e}-03$ & $2.4841 \mathrm{e}-03$ & $2.4658 \mathrm{e}-03$ \\
\hline
\end{tabular}




\section{Definition 1 [19]}

Given two minimum bounding rectangles $\mathrm{P}$ and $\mathrm{Q}$, a lower bound of the Hausdorff distance from the elements confined by $\mathrm{P}$ to the elements confined by $\mathrm{Q}$ is defined as

$$
\operatorname{HausDistLB}(P, Q)=\operatorname{Max}\left\{\begin{array}{l}
\operatorname{MinDist}\left(f_{\alpha}, Q\right): \\
f_{\alpha} \in \text { FacesOf }(P)
\end{array}\right\}
$$

Example 1 [20]

$$
\frac{\partial \tilde{U}}{\partial t}(x, t)=4 \frac{\partial^{2} \tilde{U}}{\partial x^{2}}(x, t), \quad 0<x<1, t>0
$$

with $\quad \tilde{k}[\alpha]=[\underline{k}(\alpha), \bar{k}(\alpha)]=[0.50 \alpha+0.50,1.50-0.50 \alpha]$ and subject to the boundary conditions and initial condition

$$
\begin{aligned}
& \tilde{U}(0, t)=\tilde{U}(1, t)=0, \quad t>0 \\
& \tilde{U}(x, 0)=\tilde{f}(x)=\frac{2}{\pi} \tilde{K} \sin \pi x
\end{aligned}
$$

The exact solution for

$$
\begin{aligned}
& \frac{\partial \underline{U}}{\partial t}(x, t ; \alpha)=4 \frac{\partial^{2} \underline{U}}{\partial x^{2}}(x, t ; \alpha), \\
& \frac{\partial \bar{U}}{\partial t}(x, t ; a)=4 \frac{\partial^{2} \bar{U}}{\partial x^{2}}(x, t ; a),
\end{aligned}
$$

are

$$
\begin{aligned}
& \underline{U}(x, t ; \alpha)=\frac{2}{\pi} \underline{k}(\alpha) e^{-4 \pi^{2} t} \sin \pi x, \\
& \bar{U}(x, t ; \alpha)=\frac{2}{\pi} \bar{k}(\alpha) e^{-4 \pi^{2} t} \sin \pi x
\end{aligned}
$$

respectively.

\section{Example 2 [7]}

$$
\frac{\partial \tilde{U}}{\partial t}(x, t)=\frac{\partial^{2} \tilde{U}}{\partial x^{2}}(x, t), \quad 0<x<1, t>0
$$

where $\quad \tilde{k}[\alpha]=[\underline{k}(\alpha), \bar{k}(\alpha)]=[0.50 \alpha+0.50,1.50-0.50 \alpha]$ and subject to the boundary conditions and initial condition

$$
\begin{aligned}
& \tilde{U}(0, t)=\tilde{U}(l, t)=0, \quad t>0 \\
& \tilde{U}(x, 0)=\tilde{f}(x)=\tilde{K} \sin \pi x, \quad 0 \leq x \leq 1
\end{aligned}
$$

The exact solution for

$$
\begin{aligned}
& \frac{\partial \underline{U}}{\partial t}(x, t ; \alpha)=\frac{\partial^{2} \underline{U}}{\partial x^{2}}(x, t ; \alpha), \\
& \frac{\partial \bar{U}}{\partial t}(x, t ; a)=\frac{\partial^{2} \bar{U}}{\partial x^{2}}(x, t ; a),
\end{aligned}
$$

are

$$
\begin{aligned}
& \underline{U}(x, t ; \alpha)=\underline{k}(\alpha) e^{-\pi^{2} t} \sin \pi x, \\
& \bar{U}(x, t ; \alpha)=\bar{k}(\alpha) e^{-\pi^{2} t} \sin \pi x,
\end{aligned}
$$

respectively.

From the analyses of all experimental findings by imposing the iterative methods of AGE, FSTAGE, and HSTAGE, it is apparent that the number of iterations for HSTAGE approach has decreased by approximately $71.60-72.95 \%$ relative to the FSTAGE and AGE methods. Additionally, with regard to execution time, the implementation of HSTAGE method is between $74.05-86.42 \%$ better than the FSTAGE and AGE methods. This implies that the HSTAGE technique requires the least amount of iteration counts and computing time as relative to the FSTAGE and AGE iterative approaches.

\section{Conclusions}

Herein, the families of the TAGE technique were utilized in order to resolve linear system structure by discretizing 1D-FD using a finite difference scheme. The outcomes indicate that HSTAGE technique is much better compared with FSTAGE and AGE methods, concerning iteration counts, time taken (in seconds), and Hausdorff distance. Ever since TAGE is fit adapted for parallel computation, this approach has been seen as the main advantage as it consumes sets of independent tasks that can be executed at the same time. The potential of the recommended technique is expected to be useful for the advanced exploration in deciphering any multi-dimensional FPDEs [20,21]. The outcomes of this work can basically be categorized as one of the half-sweep procedure. In order to accelerate the convergence rate of the ordinary suggested iterative approaches, a further examination on quarter-sweep techniques [22-25] may as well be taken into account in addition to the theory of the half-sweep practice.

\section{Acknowledgements}

The National Defence University of Malaysia has sponsored this research study.

\section{REFERENCES}

[1] S. L. Chang, L. A. Zadeh, On fuzzy mapping and control, IEEE Transactions on Systems, Man and Cybernatics, 1, pp. 30-34, 1972.

[2] D. Dubois, H. Prade, Towards fuzzy differential calculus part 3: differentiation, Fuzzy Sets and System, 8, pp. 225-233, 
1982.

[3] O. Kaleva, Fuzzy differential equations, Fuzzy Sets and System, 24, pp. 301-317, 1987.

[4] O. Kaleva, The Cauchy problem for fuzzy differential equations, Fuzzy Sets and System, 35, pp. 389-396, 1990.

[5] M. L. Xiao, J. Jun, H. Ling, A numerical method to solve a fuzzy differential equation via differential inclusions, Fuzzy Sets and Systems, 404, 38-61, 2021.

[6] S. Seikkala, On the fuzzy initial value problem, Fuzzy Sets and Systems, 24, pp. 319-330, 1987.

[7] T. Allahviranloo, Difference methods for fuzzy partial differential equations, Computer Methods Applied Mathematics, 2, pp. 233-242, 2002

[8] T. Allahviranloo, M. Afshar, Difference method for solving the fuzzy parabolic equations, Applied Mathematics Sciences, 1, pp. 1299-1309, 2007.

[9] D. J. Evans, M. S. Sahimi, "The Alternating Group Explicit (AGE) iterative method to solve parabolic and hyperbolic partial different equations," in Annual Review of Numerical Fluids Mechanics and Heat Transfer II, C. L. Tien and T. C. Chawla eds, Hemisphere Publ. Co., USA, 1989.

[10] K. S. Sukon, D. J. Evans, Two parameter AGE (TAGE) method for the solution of a tridiagonal linear system of equations, International Journal of Computer Mathematics, 60, pp. 265-278, 1996.

[11] A. A. Dahalan, J. Sulaiman. Implementation of TAGE method using Seikkala derivatives applied to two-point fuzzy boundary value problems, International Journal of Differential Equations, 3, 2015.

[12] R. K. Mohanty, M. K. Jain, D. Dhall, A cubic spline approximation and application of TAGE iterative method for the solution of two point boundary value problems with forcing function in integral form, Applied Mathematical Modelling, 35, pp. 3036-3047, 2011.

[13] N. Jha, R. K. Mohanty, TAGE iterative algorithm and nonpolynomial spline basis for the solution of nonlinear singular second order ordinary differential equations, Applied Mathematics and Computation, 218, pp. 3289-3296, 2011.

[14] A. R. Abdullah, The four-point Explicit Decoupled Group (EDG) method: A fast Poisson solver, International Journal Computer Mathematics, 38, pp. 61-70, 1991.
[15] M. Othman, A. R. Abdullah, An efficient four points Modified Explicit Group Poisson solver, International Journal Computer Mathematics, 76, 2, pp. 203-217, 2000.

[16] R. K. Mohanty, D. Dhall, Third order accurate variable mesh discretization and application of TAGE iterative method for the non-linear two-point boundary value problems with homogeneous functions in integral form, Applied Mathematics and Computation, 215, pp. 2024-2034, 2009.

[17] R. K. Mohanty, N. Khosla, Application of TAGE iterative algorithms to an efficient third order arithmetic average variable mesh discretization for two-point non-linear boundary value problems, Applied Mathematics and Computation, 172, pp. 148-162, 2006.

[18] K. S. Sukon, On two parameter alternating group explicit (TAGE) method for singular perturbation problems, Parallel Algorithms Applied, 10, pp. 71-77, 1996.

[19] S. Nutanog, H. E. Jacox, H. Samet, An incremental Hausdorff distance calculation algorithm, Proceedings VLDB Endowment, 4, pp. 506-517, 2011.

[20] A. Farajzadeh, A. H. Pour, M. Amini, An explicit method for solving fuzzy partial differential equation, International Mathematic Forum, 5, pp. 1025-1036, 2010.

[21] F. Mirzaee, M. K. Yari, A novel computing three-dimensional differential transform method for solving fuzzy partial differential equations, Ain Shams Engineering Journal, 7, 2, 695-708, 2016.

[22] J. C. V. Lung, J. Sulaiman, On a quarter-sweep finite difference scheme for one-dimensional porous medium equations, International Journal of Applied Mathematics, 33, 3 , pp. 439-450, 2020.

[23] J. H. Eng, A. Saudi, J. Sulaiman, Numerical evaluation of quarter-sweep SOR iteration for solving poisson image blending problem, in Proceedings - 2018 IEEE International Conference on Artificial Intelligence in Engineering and Technology, IICAIET2018, 8638469, pp. 27-31, 2019.

[24] A. A. Dahalan, A. Saudi, J. Sulaiman, Numerical technique for autonomous robot path planning based on QSAOR iterative method using indoor environment, Journal of Engineering and Applied Sciences, 13, 20, pp. 8414-8418, 2018.

[25] A. A. Dahalan, N. S. A. Aziz, J. Sulaiman, QSAGE iterative method for the numerical solution of two-point fuzzy boundary value problem, Journal of Engineering and Applied Sciences, 12, 16, pp. 4068-4074, 2017. 